Check for updates

Cite this: RSC Adv., 2017, 7, 52555

\section{A simple and efficient approach to fabricate graphene/CNT hybrid transparent conductive films}

\begin{abstract}
Jianhua Zhang, Zhangfu Chen, Xiaoxue Xu, Wei Liao and Lianqiao Yang (D)*
In this paper, a novel and scalable method to fabricate graphene/carbon nanotube (CNT) hybrid transparent conductive films on Cu substrates, which combines electroplating and chemical vapor deposition (CVD) is proposed and demonstrated. The $\mathrm{Cu}$ substrate was electroplated with electrolyte containing conductive CNTs; then, a uniform graphene film was grown on $\mathrm{Cu}$. After a commonly utilized polymethyl methacrylate assisted transfer process, a hybrid graphene/CNT transparent conductive film was obtained at the target substrate. Conventional graphene grown on electropolished $\mathrm{Cu}$ was used as the reference sample. The comprehensive characterization using scanning electron microscopy (SEM), Raman microscopy system, and transmission electron microscopy (TEM) selected area electron diffraction pattern show that the CNTs are uniformly covered by a monolayer graphene with comparable quality to graphene grown on electropolished $\mathrm{Cu}$. The hybrid thin films exhibit outstanding surface morphology (RMS of 1.26), obviously enhanced electrical properties (the square resistance decreases from 490 to $394 \Omega \mathrm{sq}^{-1}$ ), better surface wettability ( $7^{\circ}$ decrease in contact angle), and a negligible transmittance loss ( $1.3 \%$ reduction at $550 \mathrm{~nm}$ ) compared to CVD graphene that was grown on electropolished $\mathrm{Cu}$. It is anticipated that the graphene/CNT hybrid films that are fabricated using the proposed approach can be a promising alternative to ITO to realize the emerging, particularly flexible optoelectronic devices.
\end{abstract}

Received 4th September 2017 Accepted 7th November 2017

DOI: 10.1039/c7ra09809j

rsc.li/rsc-advances one-dimensional CNT, many researchers have devoted to fabricate hybrid nanostructures and attempted their applications in optoelectronic devices. ${ }^{1 \mathbf{1 1 4}}$ For example, Tung et al. demonstrated a hybrid nanostructure of reduced graphene oxide (rGO) and CNT for transparent conductors. ${ }^{11}$ Using the chemical vapor deposition (CVD) method, Qiu et al. prepared a nanocomposite, where freestanding aligned CNT arrays grew on a large-area single-layered graphene sheet. ${ }^{12}$ Wang et al. fabricated three-dimensional fewlayer graphene and CNT foam architectures. ${ }^{13}$ Using a two-step CVD method, Dong et al. synthesized the 3D graphene/CNT hybrid structures to apply to electrochemical sensing. ${ }^{14}$ However, these hybrid nanostructures either use non-ideal rGO as the substitute of graphene or require complex fabrication processes. In addition, the CNTs are usually spin-coated or printed onto the target substrate and physically attached to graphene, which cannot offer an effective electron transport network between CNT and graphene.

Here, this paper reports a simple method to form graphene/ CNT hybrid thin films using one-step CVD growth on a Cu foil substrate pre-electroplated with conductive CNT. The performance of hybrid TCF is significantly enhanced, and the possible reasons of this enhancement are explored.

\section{Experimental}

\subsection{Synthesis of graphene/CNT hybrid films}

In this paper, graphene grown on electropolished and electroplated $\mathrm{Cu}$ foils were used as the reference samples. Graphene
Key Laboratory of Advanced Display and System Applications, Ministry of Education Shanghai University, Yanchang Road 149, Shanghai 200072, China. E-mail: yanglianqiao@i.shu.edu.cn 
fabricated on electropolished $\mathrm{Cu}$ is named graphene ${ }_{(\mathrm{a})}$ because the electropolished $\mathrm{Cu}$ is the anode of the electrolytic solution. Similarly, graphene fabricated on electroplated $\mathrm{Cu}$ is named $\operatorname{graphene}_{(\mathrm{c})}$. The pre-treatment of the $\mathrm{Cu}$ foil, fabrication and transfer process of graphene are shown below.

2.1.1 Pre-treatment of the $\mathrm{Cu}$ foil. Graphene sheets were fabricated via low-pressure CVD using copper foil $(25 \mu \mathrm{m}$ thick, $99.8 \%$, Alfa Aesar) as a metal catalyst. Before graphene growth, the received $\mathrm{Cu}$ foils, which were cut with size of $3 \times 2.5 \mathrm{~cm}^{2}$, were electropolished at the voltage of $7 \mathrm{~V}$ for $90 \mathrm{~s}$. The electrolyte was composed of $100 \mathrm{~mL}$ of water, $50 \mathrm{~mL}$ of orthophosphoric acid, $50 \mathrm{~mL}$ of ethanol, $10 \mathrm{~mL}$ of isopropyl alcohol, and $1 \mathrm{~g}$ of urea. After electro-polishing, the $\mathrm{Cu}$ foil was rinsed with deionized water, washed with acetone and ethanol, and blowdried with nitrogen.

2.1.2 Fabrication process of graphene. The electropolished $\mathrm{Cu}$ foils were placed in the CVD furnace, and the chamber was evacuated to a base pressure of 40-70 mTorr. Next, the system was heated to a growth temperature of $1000{ }^{\circ} \mathrm{C}$ under a mixed gas $\left(\mathrm{H}_{2}: \mathrm{Ar}=1: 9\right)$ flow of $100 \mathrm{sccm}$ ( $\left.\sim 650 \mathrm{mTorr}\right)$ and annealed for 30 minutes. Then, $5 \mathrm{sccm}$ acetylene was introduced for 15 minutes at a total pressure of 700 mTorr. After growth, the samples were cooled to room temperature in the mixed gas (without acetylene) flow of $40 \mathrm{sccm}$.

2.1.3 Transfer process of graphene. The polymethyl methacrylate (PMMA, Sigma-Aldrich, dissolved in chlorobenzene with a concentration of $40 \mathrm{mg} \mathrm{mL}^{-1}$ ) was spin-coated at $2000 \mathrm{rpm}$ for $60 \mathrm{~s}$ on the as-grown graphene film and cured at $180{ }^{\circ} \mathrm{C}$ for 1 minute on a hot plate. Argon plasma ( $9 \mathrm{~W}, 3 \mathrm{~min}$ ) was used to remove the backside graphene of $\mathrm{Cu}$ foil. Then, the $\mathrm{Cu}$ substrate was etched away by Marble's reagent. The graphene/PMMA films were rinsed with deionized water and placed on the target substrates. The protective PMMA was removed by acetone after the transferred graphene film completely dried.

2.1.4 Fabrication process of the graphene/CNT hybrid film. The graphene/SWCNT hybrid films were synthesized by fabricating graphene on a copper foil, which was pre-electroplated with SWCNT. The composition of the electrolyte was $100 \mathrm{~mL}$ of water, $50 \mathrm{~mL}$ of orthophosphoric acid, $50 \mathrm{~mL}$ of ethanol, $10 \mathrm{~mL}$ of isopropyl alcohol, $1 \mathrm{~g}$ of urea, and $10 \mathrm{~mL}$ of $95 \%$ metallic SWCNT solution (XF Nano Ltd.) with a concentration of $0.01 \mathrm{mg} \mathrm{mL} \mathrm{m}^{-1}$. The pre-cleaned $\mathrm{Cu}$ foils were electroplated in the solution with the voltage of $7 \mathrm{~V}$ for $90 \mathrm{~s} .{ }^{15}$ Then, the identical CVD deposition and transfer process were performed to fabricate graphene on CNT-coated $\mathrm{Cu}$.

\subsection{Characterization}

The nanostructure and surface morphologies of the hybrid films were recorded using a scanning electron microscope (SEM, HITACHI 4800). The electrical properties of the TCFs were obtained using a Hall Measurement System (ACCENT HL5550LN2). The optical properties were measured with UVvisible spectra $(\mathrm{U}-3900 \mathrm{H})$, and the surface morphologies were tested using an atomic force microscope (AFM, SPA-400 SPM). The Raman spectra were evaluated with a Raman microscopy system (LabRAM HR XploRA) at a laser wavelength of $532 \mathrm{~nm}$. The selected area electron diffraction pattern of graphene was characterized using a transmission electron microscope (TEM, FEI Talos). The contact angle was measured by a Contact Angle Instrument (Vino, SL200KS). For the transmission measurement, the hybrid film was transferred onto the glass substrate with a sample size of $2 \times 2 \mathrm{~cm}^{2}$. With the exception of the transmission measurement, all measurements, including SEM, Hall test, AFM, Raman, wettability test, were obtained with the hybrid film transferred onto the $\mathrm{SiO}_{2} / \mathrm{Si}$ substrate with a sample size of $1 \times 1 \mathrm{~cm}^{2}$.

\section{Results and discussions}

The graphene/SWCNT hybrid TCFs were synthesized by growing graphene using CVD on the copper foil pre-electroplated with SWCNT. The electroplating processes of SWCNT is schematically illustrated in Fig. 1. The processes can be divided into five steps. First, the surface of the SWCNT was adsorbed by numerous $\mathrm{Cu}^{2+}$ ions, most of which located at the ends of the SWCNT, and therefore obtained a $\mathrm{Cu}^{2+} /$ SWCNT complex in the electrolyte. ${ }^{\mathbf{1 6 , 1 7}}$ Second, after the voltage of $7 \mathrm{~V}$ was applied, a current of $1 \mathrm{~A}$ was obtained, and the $\mathrm{Cu}^{2+}$ /SWCNT complex moved toward the cathode $\mathrm{Cu}$ foil under the action of electrophoresis. ${ }^{18,19}$ Third, when the $\mathrm{Cu}^{2+} /$ SWCNT complex reached the cathode and formed a weak adsorption at the surface, two reduction steps were accomplished: from $\mathrm{Cu}^{2+}$ to $\mathrm{Cu}^{+}$and subsequently from $\mathrm{Cu}^{+}$to $\mathrm{Cu}$. The reduced $\mathrm{Cu}$ particles can be obtained on the surface of the cathode $\mathrm{Cu}$ foil. Because of the presence of metallic SWCNT, the electrical conductivity of the electroplating solution improved; then, the reduction reaction from $\mathrm{Cu}^{2+}$ to $\mathrm{Cu}^{+}$was obviously promoted. Fourth, as increasingly more $\mathrm{Cu}^{2+} / \mathrm{SWCNT}$ complexes reached the cathode surface, either end of the $\mathrm{Cu}^{2+} / \mathrm{SWCNT}$ complex inserted into cathode $\mathrm{Cu}$ foil surface, where the reduction reaction was processing from $\mathrm{Cu}^{+}$to $\mathrm{Cu}$ particles, which resulted in the SWCNT/ $\mathrm{Cu}$ hybrid structures on the surface of the $\mathrm{Cu}$ foil. Finally, the SWCNT-coated cathode $\mathrm{Cu}$ foil was rinsed and blow-dried with pure nitrogen, which made the SWCNT partly inserted in the $\mathrm{Cu}$

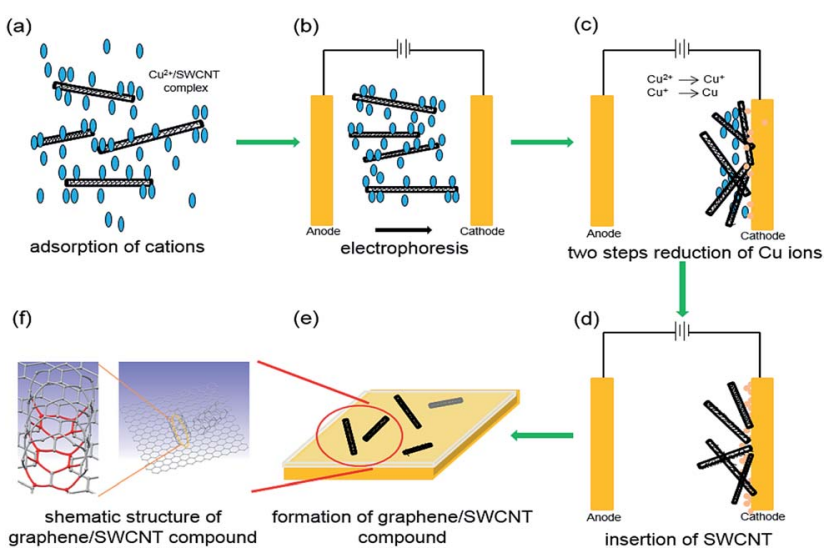

Fig. 1 Schematic of fabrication processes for graphene/SWCNT hybrid TCF. 


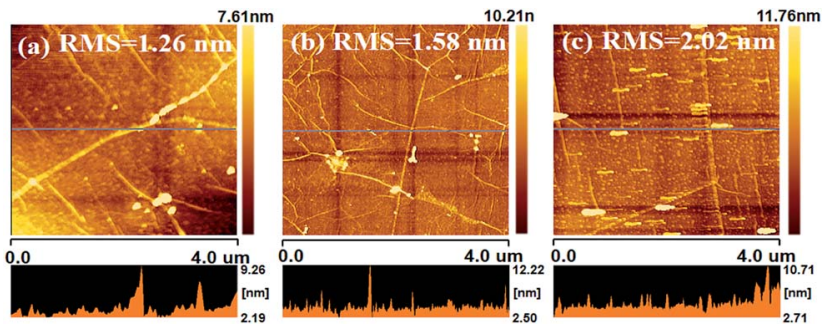

Fig. 2 Surface roughness of (a) SWCNT/graphene hybrid thin films, (b) graphene $_{(\mathrm{a})}$, (c) graphene $(\mathrm{c})$, transferred on $\mathrm{Si} / \mathrm{SiO}_{2}$ substrate.

foil and almost horizontally distributed on the surface of $\mathrm{Cu}$ foil. Then, the CVD process was performed to synthesize SWCNT/graphene hybrid thin films. Compared with the spincoating or printing method, the CNT was partly embedded in the $\mathrm{Cu}$ foil, which acted as the catalyst of graphene and might form chemical bonding between CNT and graphene.

The surface condition is an important factor to elucidate the quality of graphene, including the pre-treatment, fabrication and transfer processes. Fig. 2 shows the surface morphology of three types of TCFs. The SWCNT with a diameter of approximately $3 \mathrm{~nm}$ is clearly observed in Fig. 2a. It is easy to understand that the $\mathrm{Cu}$ on the anode has a smoother surface and less impurities than that on cathode, and the surface morphology of the $\mathrm{Cu}$ substrate is directly proportional to the graphene surface condition. However, the root-mean-square (RMS) of hybrid TCF is only approximately $1.26 \mathrm{~nm}$, which is lower than graphene $e_{(\mathrm{c})}$ $(1.58 \mathrm{~nm})$ and graphene $($ a) $(2.02 \mathrm{~nm})$. The good surface morphology of hybrid films likely indicates that the SWCNT acted as a "filler" and flattened the rugged Cu surface, thereby increasing the flat level of the catalyst in the CVD process. The SWCNT also played the role of a "soft" buffer that decreased the mismatch in surface morphology between the soft $\mathrm{Cu}$ substrate and the rigid $\mathrm{SiO}_{2} / \mathrm{Si}$ substrate in the transfer process. Thus, a low surface roughness and a small value of RMS on the hybrid films were obtained. ${ }^{20}$ In order to evaluate the thickness of hybrid film, an artificial scratch was made and the thickness was found to be about $0.8 \mathrm{~nm}$ as shown in Fig. 3a and b. The improvement in RMS can be more useful and applicable for its utilization in organic optoelectronic devices because they commonly require good surface morphology.

To further investigate the surface condition, SEM images of graphene/SWCNT hybrid TCFs on the $\mathrm{SiO}_{2} / \mathrm{Si}$ substrate were captured, and the results are shown in Fig. 4a. Fig. 4a shows that SWCNT was perfectly wrapped by the graphene layer, and
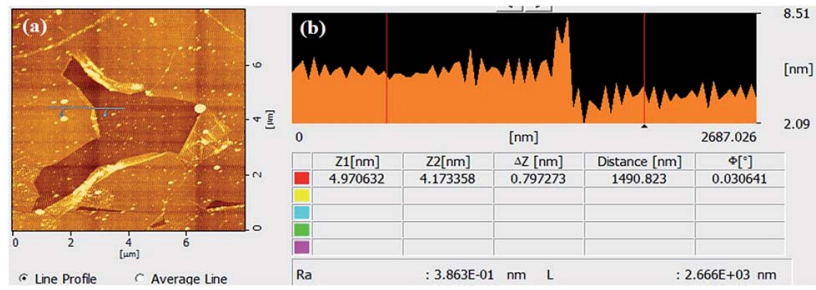

Fig. 3 (a) AFM image of SWCNT/graphene hybrid thin films with an artificial scratch, (b) profile plot of the height along the line in (a). no damage was observed on the SWCNT, which demonstrates that SWCNT remains stable during the electroplating and CVD processes. Furthermore, no impurities and defects such as wrinkles, folds, cracks and ripples were observed on the graphene layer of hybrid TCF, which also indicates that the TCFs possess a good surface morphology.

Raman spectroscopy, which is a typical and effective method to evaluate the structure and defects of carbon-based lowdimensional materials, was used to evaluate the TCF, and the results are shown in Fig. 4 b. Fig. $4 \mathrm{~b}$ displays the average Raman spectra of 5 points in TCF with excitation wavelength of $532 \mathrm{~nm}$ on the $\mathrm{SiO}_{2} / \mathrm{Si}$ substrate. The low D peak at $\sim 1350 \mathrm{~cm}^{-1}$ of graphene, which corresponds to the degree of crystallinity and defect, indicates that the CVD graphene has high quality. The two prominent peaks at $\sim 1570$ and $2700 \mathrm{~cm}^{-1}$ can correspond to the $\mathrm{G}$ and $2 \mathrm{D}$ bands, respectively. ${ }^{21}$ The $\mathrm{G}$ band is created by the $\mathrm{E}_{2 \mathrm{D}}$ phonon at the central area of Brillouin zone, and the 2D band represents double-resonant Raman scattering. ${ }^{22}$ The peak ratio of $\mathrm{G} / 2 \mathrm{D}$ is 0.85 , and the symmetric $2 \mathrm{D}$ band is centered at $2701 \mathrm{~cm}^{-1}$ with a full width at half maximum of $33 \mathrm{~cm}^{-1}$, which demonstrates that the graphene sheet is monolayer dominated. ${ }^{23,24}$ The intensities of the $\mathrm{G}$ and $2 \mathrm{D}$ band peaks of SWCNT are distinctly higher than those of the graphene film possibly because of the SWCNT has a significantly stronger scatter signal. For the hybrid TCFs, the peak G/2D ratio of 0.65 is between those of SWCNT (0.35) and graphene (0.85), which indicates the presence of both CNT and graphene. In addition, the peak at $\sim 2450$ corroborates the combination of CNT and

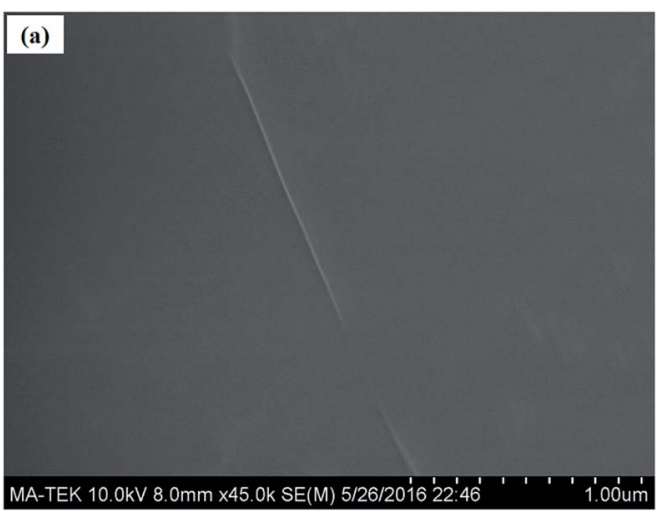

(b)

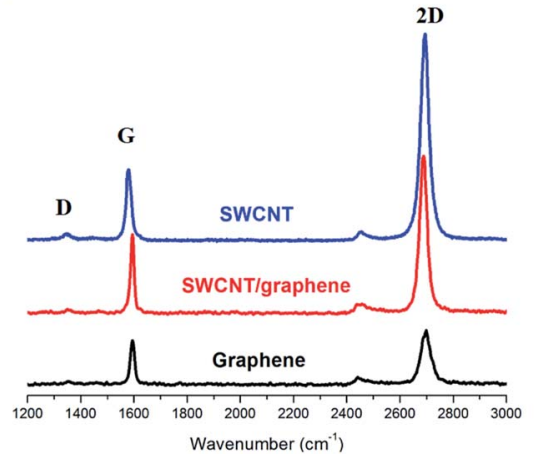

Fig. 4 (a) SEM and (b) Raman spectra of TCFs on $\mathrm{SiO}_{2} / \mathrm{Si}$ substrate at laser excitation wavelength of $532 \mathrm{~nm}$. 
graphene, although its interpretation was still subject to debate. $^{25}$

To further confirm the crystallinity and the layers of graphene, SAED was conducted on the graphene transferred to TEM grid and the results are shown in Fig. 5. The clear diffraction pattern (Fig. 5a) identifies the good crystallinity of graphene. The line profile of the diffraction patterns (Fig. 5b) demonstrates the monolayer nature of the graphene domain.

Optical transmittance is an important factor for TCFs. Fig. 6a shows the optical transmittance spectra of TCFs transferred to glass substrates. The optical transmittance of graphene $(\mathrm{c})$ was $96.5 \%$, which was slightly lower than that of graphene $(a)(97.2 \%)$ at the wavelength of $550 \mathrm{~nm}$. The transmittance difference was mainly caused by the surface impurities of $\mathrm{Cu}$ at the anode and cathode. In contrast, the transmittance of hybrid TCF was 95.9\%, which is slightly lower than the two others as anticipated. However, the transmittance of hybrid TCF remains higher than $94 \%$ in the visible light range of $400-800 \mathrm{~nm}$, whose performance is more than acceptable for its application as TCF. The inset graph of Fig. 6a shows the scene through the SWCNT/ graphene hybrid film on glass; the red dotted rectangular shows the area of the sample with the size of $2 \times 2 \mathrm{~cm}^{2}$. No vision disparity is observed in the graph, which also confirms the uniformity and transparency of the hybrid films.

As an alternative of ITO, the electrical conductivity is the foremost issue of TCF in addition to the optical performance and surface condition. The sheet resistance of three types of TCFs that were transferred onto the $\mathrm{SiO}_{2} / \mathrm{Si}$ substrates was measured

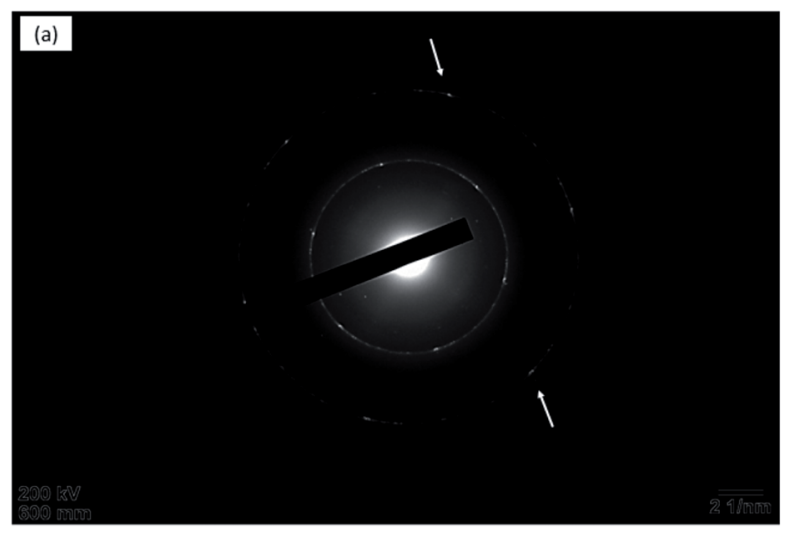

(b)

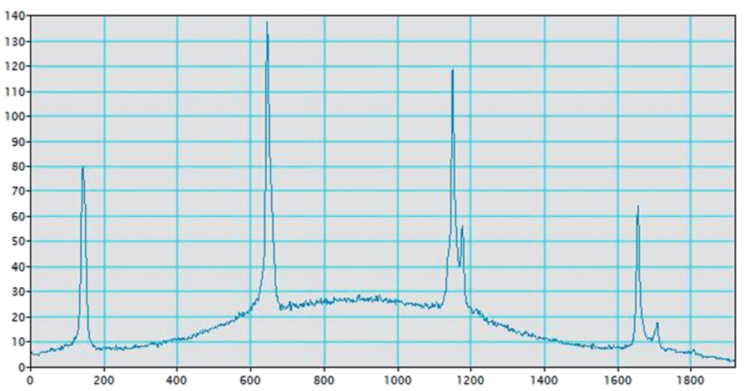

Fig. 5 (a) SAED pattern of graphene, (b) profile plots of the diffraction peak intensities along the arrows in (a). (a)

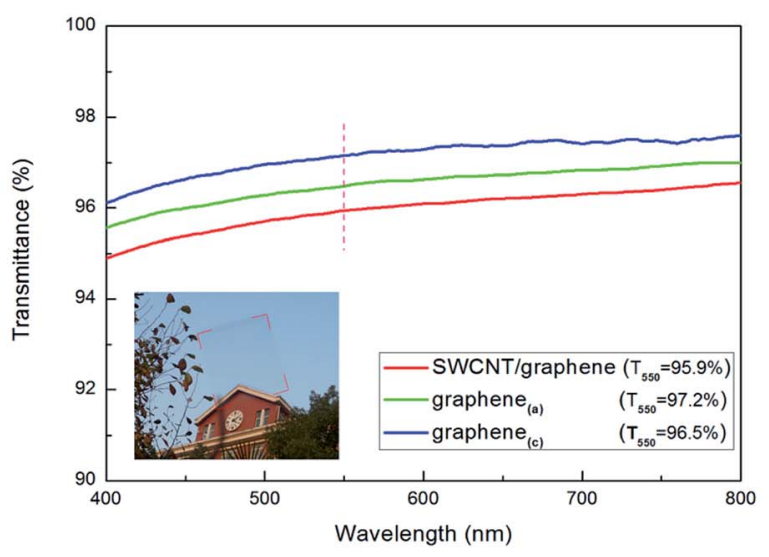

(b)

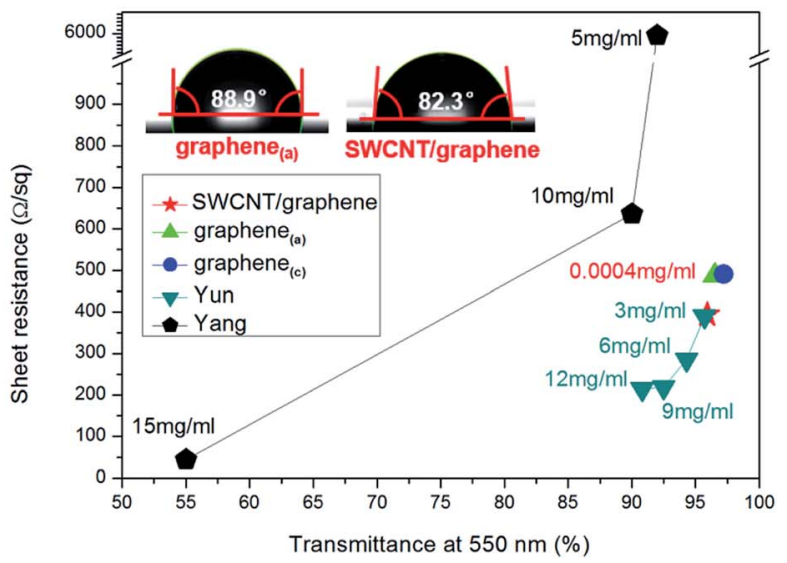

Fig. 6 (a) Optical transmittance spectra of TCF. The inset graph is the scene through the SWCNT/graphene hybrid film. (b) Sheet resistance versus optical transmittance of TCF, the inset graphs are the water contact angel results of graphene ${ }_{(a)}$ and SWCNT/graphene.

by Hall effect measurement system, and the results are shown in Fig. 6b. Fig. $6 \mathrm{~b}$ shows that the sheet resistance of the hybrid TCF is $394 \Omega \mathrm{sq}^{-1}$ with a transmittance of $95.9 \%$ at the wavelength of $550 \mathrm{~nm}$, which is significantly lower than those of graphene ${ }_{(a)}$ and graphene ${ }_{(\mathrm{c})}$. In particular, few numbers of SWCNT from Fig. 6a can distinctly decrease the sheet resistance of the SWCNT/ graphene hybrid films, which confirms that few SWCNTs can add conductive channels and repair line defects to decrease the electrical resistance of CVD-grown graphene. The graphene/ SWCNT hybrid films were investigated by Yun and Yang, and it has been shown that the decrease in sheet resistance significantly depends on the CNT concentration. ${ }^{11,26}$ In this study, the concentration of the SWCNT solution is $0.0004 \mathrm{mg} \mathrm{mL}^{-1}$, which is far below the concentration in the literature with the identical range of transmittance and sheet resistance. In other words, the electroplating method of the CNT to the $\mathrm{Cu}$ substrate shows better enhancement effect than the commonly used approach to fabricate graphene/CNT hybrid TCF. For the CNTs that are spincoated or printed on the target substrate, the physical adhesion of CNT to graphene cannot form effective chemical bonding, 
which hinders the carrier transport between CNT and graphene. In this study, the CNT was partially embedded in $\mathrm{Cu}$, which acted as the catalyst of graphene; during the deposition process, the chemical bond might form between graphene and CNT. Thus, the contact resistance between graphene and CNT significantly decreased; then, the carrier transport was enhanced compared to conventional methods. Because of the extremely low concentration of the SWCNT solution, good balance between sheet resistance and transmittance for the nanoscale thickness TCF was obtained and further demonstrates that the possible formation of the chemical bond between graphene and CNT strengthens the carrier transport and conductivity.

The wetting properties of TCF to function materials directly affect the final application in optical devices. The Contact Angle Instrument was used to evaluate the wetting performance, and the results are shown in the inset graphs of Fig. 6b. The water contact angles (WCAs) of graphene ${ }_{(a)}$ and SWCNT/graphene are $88.9^{\circ}$ and $82.3^{\circ}$, respectively, which indicates that SWCNT/ graphene is more hydrophilic than graphene $e_{(a)}$. The wetting property of graphene-based TCF is a widely concerned issue, and the WCAs vary from $\sim 40^{\circ}$ to $\sim 90^{\circ}$ in the literature..$^{27,28}$ It has been widely accepted that the WCA is related to the physicochemical composition, surface roughness, surface functional groups and doping level of graphene-based TCF, which eliminates the equipment error. ${ }^{28}$ Here, we would not strive to distinguish the specific reason of hydrophilic improvement because it is not the main point of this study. Instead, we would like to emphasize that the increase in wetting property of SWCNT/graphene can be helpful in the application of transparent conductive electrodes in optoelectronic devices.

In this study, we only investigated the SWCNT at a certain concentration with the electroplating target of $\mathrm{Cu}$ and corroborated the quality enhancement effect of hybrid TCF. In fact, we believe that the proposed approach is applicable to any conductive CNT and graphene catalyst as long as it requires electro-polishing pre-treatment.

\section{Conclusions}

In this paper, graphene/SWCNT hybrid TCFs were synthesized by growing graphene on $\mathrm{Cu}$ foil using $\mathrm{CVD}$, which was preelectroplated with conductive SWCNT. The fabricated hybrid film using the proposed approach is proven to have better electrical conductance, better surface morphology and negligible loss in transmittance compared with that grown on an electropolished $\mathrm{Cu}$ catalyst. Compared with conventional fabrication approaches of graphene/CNT hybrid TCF, much better optelectrical performance is obtained at the identical concentration of CNT solution. In addition, the increase in wetting property of SWCNT/graphene can be helpful in the application of TCFs in optoelectronic devices. We have proposed and proven that graphene/SWCNT hybrid thin films fabricated by the one-step CVD growth on the $\mathrm{Cu}$ foil substrate, which has been preelectroplated with conductive CNT, is a simple and effective method to obtain high-quality hybrid TCFs.

\section{Conflicts of interest}

There are no conflicts to declare.

\section{Acknowledgements}

This work is financially supported by the National Natural Science Foundation of China (51505270) and the Science and Technology Committee of Shanghai (15590500500).

\section{References}

1 K. Kwak, K. Cho and S. Kim, Sci. Rep., 2013, 3, 2787-2793.

2 S. Chen, L. Deng, J. Xie, L. Peng, L. Xie, Q. Fan and W. Huang, Adv. Mater., 2010, 22, 5227-5239.

3 W. Fu, L. Liu, K. Jiang, Q. Li and S. Fan, Carbon, 2010, 48, 1876-1879.

4 D. Hecht, L. Hu and G. Grüner, Appl. Phys. Lett., 2006, 89, 425-428.

5 Z. Hu, J. Zhang, Z. Hao, Q. Hao, X. Geng and Y. Zhao, Appl. Phys. Lett., 2011, 98, 66-69.

6 K. S. Novoselov, A. K. Geim, S. V. Morozov, D. Jiang, Y. Zhang, S. V. Dubonos, I. V. Grigorieva and A. A. Firsov, Science, 2004, 306, 666-669.

7 K. I. Bolotin, K. J. Sikes, Z. Jiang, M. Klima, G. Fudenberg, J. Hone, P. Kim and H. L. Stormer, Solid State Commun., 2008, 146, 351-355.

8 X. Dong, Y. Shi, Y. Zhao, D. Chen, J. Ye, Y. Yao, F. Gao, Z. Ni, T. Yu, Z. Shen, Y. Huang, P. Chen and L. J. Li, Phys. Rev. Lett., 2009, 102, 135501-213554.

9 K. S. Kim, Y. Zhao, H. Jang, S. Y. Lee, J. M. Kim, K. S. Kim, J. H. Ahn, P. Kim, J. Y. Choi and B. H. Hong, Nature, 2009, 457, 706-710.

10 Y. Fu, B. Carlberg, N. Lindahl, N. Lindvall, J. Bielecki, A. Matic, Y. Song, Z. Hu, Z. Lai, L. Ye, J. Sun, Y. Zhang, Y. Zhang and J. Liu, Adv. Mater., 2012, 24, 1576-1581.

11 V. C. Tung, L. M. Chen, M. J. Allen, J. K. Wassei, K. Nelson, R. B. Kaner and Y. Yang, Nano Lett., 2009, 9, 1949-1955.

12 L. Qiu, Q. Wu, Z. Yang, X. Sun, Y. Zhang and H. Peng, Small, 2015, 11, 1150-1155.

13 W. Wang, S. Guo, M. Penchev, I. Ruiz, K. N. Bozhilov, D. Yan, M. Ozkan and C. S. Ozkan, Nano Energy, 2013, 2, 294-303.

14 X. Dong, Y. Ma, G. Zhu, Y. Huang, J. Wang, M. B. Chan-Park, L. Wang, W. Huang and P. Chen, J. Mater. Chem., 2012, 22, 17044-17048.

15 L. Wang, W. Feng, L. Yang and J. Zhang, Acta Phys. Sin., 2014, 2014(63), 176801.

16 E. Bezus, H. Brown and L. Tomaszewski, Plating, 1969, 56, 1234-1239.

17 C. Buelens, J. P. Celis and J. R. Roos, J. Appl. Electrochem., 1983, 13, 541-548.

18 J. Fransaer, J.-P. Celis and J. Roos, J. Electrochem. Soc., 1992, 139, 413-425.

19 N. Guglielmi, J. Electrochem. Soc., 1972, 119, 1009-1012.

20 I. N. Kholmanov, C. W. Magnuson, R. Piner, J. Y. Kim, A. E. Aliev, C. Tan, T. Y. Kim, A. A. Zakhidov, 
G. Sberveglieri, R. H. Baughman and R. S. Ruoff, Adv. Mater., 2015, 27, 3053-3059.

21 X. Dong, D. Fu, W. Fang, Y. Shi, P. Chen and L. J. Li, Small, 2009, 5, 1422-1426.

22 T. H. Seo, A. H. Park, G. H. Lee, M. J. Kim and E.-K. Suh, J. Phys. D: Appl. Phys., 2014, 47, 315102-315108.

23 L. Gao, W. Ren, J. Zhao, L. P. Ma, Z. Chen and H. M. Cheng, Appl. Phys. Lett., 2010, 97, 183-186.

24 V. P. Verma, S. Das, I. Lahiri and W. Choi, Appl. Phys. Lett., 2010, 96, 203108-203111.
25 A. C. Ferrari and D. M. Basko, Nat. Nanotechnol., 2013, 8, 235-246.

26 A. L. Gorkina, A. P. Tsapenko, E. P. Gilshteyn, T. S. Koltsova, T. V. Larionova, A. Talyzin, A. S. Anisimov, I. V. Anoshkin, E. I. Kauppinen, O. V. Tolochko and A. G. Nasibulin, Carbon, 2016, 100, 501-507.

27 R. Raj, S. C. Maroo and E. N. Wang, Nano Lett., 2013, 13, 1509-1515.

28 A. Ashraf, Y. Wu, M. C. Wang, K. Yong, T. Sun, Y. Jing, R. T. Haasch, N. R. Aluru and S. Nam, Nano Lett., 2016, 16, 4708-4712. 